\title{
Rényi entropies for multidimensional hydrogenic systems in position and momentum spaces
}

\author{
D. Puertas-Centeno $*$ I.V. Toranzo $t \pm$ and J.S. Dehesd $t$ \\ Departamento de Física Atómica, Molecular y Nuclear, \\ Universidad de Granada, Granada 18071, Spain and \\ Instituto Carlos I de Física Teórica y Computacional, \\ Universidad de Granada, Granada 18071, Spain
}

\begin{abstract}
The Rényi entropies of Coulomb systems $R_{p}[\rho], 0<p<\infty$ are logarithms of power functionals of the electron density $\rho(\vec{r})$ which quantify most appropriately the electron uncertainty and describe numerous physical observables. However, its analytical determination is a hard issue not yet solved except for the first lowest-lying energetic states of some specific systems. This is so even for the $D$-dimensional hydrogenic system, which is the main prototype of the multidimensional Coulomb many-body systems. Recently, the Rényi entropies of this system have been found in the two extreme high-energy (Rydberg) and high-dimensional (pseudo-classical) cases. In this work we determine the position and momentum Rényi entropies (with integer $p$ greater than 1) for all the discrete stationary states of the multidimensional hydrogenic system directly in terms of the hyperquantum numbers which characterize the states, the nuclear charge and the space dimensionality. We have used a methodology based on linearization formulas for powers of the orthogonal Laguerre and Gegenbauer polynomials which control the hydrogenic states.
\end{abstract}

Keywords: Rényi entropies, multidimensional hydrogenic systems, Rényi entropies of multidimensional hydrogenic systems in position space, Rényi entropies of multidimensional hydrogenic systems in momentum space, Linearization of powers of orthogonal polynomials.

\section{INTRODUCTION}

In a seminal paper Alfréd Rényi [1] found axiomatically a set of monoparametric information entropies of a probability density $\rho(\vec{x})$ which includes the Shannon entropy as a limiting case. These Rényi quantities are logarithms of integral functionals of powers of $\rho(\vec{x})$ (Yule-Sichel frequency

*vidda@correo.ugr.es

tivtoranzo@ugr.es

†dehesa@ugr.es 
moments [2 4]) appropriately renormalized to have an entropic character, as

$$
R_{q}[\rho]:=\frac{1}{1-q} \ln \int_{\mathbb{R}^{D}}[\rho(\vec{x})]^{q} d \vec{x}, \quad \vec{x} \in \mathbb{R}^{D}, q \neq 1
$$

These entropies, which completely characterize the density under certain conditions, quantify various spreading-like facets (governed by the parameter $q$ ) of the probability density $\rho(\vec{x})$, including the Shannon entropy (when $q \rightarrow 1$ ) and the disequilibrium (when $q=2$ ) which measures the separation of the distribution with respect to equiprobability. Moreover, when $q \rightarrow 0$ this quantity is proportional to the logarithm of the volume of the multidimensional support set, and when $q \rightarrow \infty$ the Rényi entropy puts more emphasis on where $\rho(\vec{x})$ attains its maximum. The parameter $q$ has different meanings depending on the context; for instance, it can be interpreted as the inverse of the temperature in thermodynamic systems and it is related to the Reynolds number in turbulence theory [5]. Moreover, the Rényi entropies are closely related to other information-theoretic quantities such as e.g., the Tsallis entropies [6] which play a very important role in systems with strong long-range correlations and nonextensive statistical mechanics [7, 8]. Furthermore, from the viewpoint of thermodynamics, the Rényi entropy its directly connected to the free energy of a system in thermal equilibrium, a relation that holds for both classical and quantum realms, and so accounts for the work that the system is capable of.

The properties of the Rényi entropies and their applications have been widely considered/applied (see e.g., [9 22]) and reviewed [23 26] in a broad variety of fields ranging from applied mathematics, quantum physics, Rydberg physics, complexity theory to non-linear physics, option price calibration, nanotechnology and neuroscience. However, these quantities have not yet been exactly calculated except for a few one-dimensional exponential densities (see e.g., [15]) and some probability densities of a single-particle system moving in the elementary multidimensional quantum potentials of infinite well [16] and rigid rotator [21]) types. Moreover, the dominant term for the Rényi entropies of the multidimensional harmonic oscillator has been determined at the high-dimensional (pseudoclassical) and high-energy (Rydberg) limits [27], and then the entropy values for both ground and excited oscillator-like states have been analytically calculated [28] in terms of the hyperquantum numbers and the oscillator strength. Here we should also mention the efforts to understand the role of quantum entanglement of many-body systems by determining the quantum Rényi entropy of one-dimensional many-particle model systems under special and limiting conditions, such as finite systems of free fermions in a continuum interval, statistical models with finite numbers of degrees of freedom, the one-dimensional XY quantum spin chain in 
a transverse magnetic field and the totally asymmetric exclusion process [29 32], among others.

Recently, the analytical determination of the Rényi entropies of the main prototype of the $D$-dimensional Coulomb many-body systems, the $D$-dimensional hydrogenic system, from first principles (i.e., in terms of the hyperquantum numbers of the state and the nuclear charge) has been undertaken [33 35]. This is relevant per se and for a reference point of view for all multidimensional Coulomb systems. The $D$-dimensional hydrogenic system is a negatively-charged particle moving in a space of $D$ dimensions around a positively charged core which electromagnetically binds it in its orbit [33, 36 39, 41, 42, 52]. This system allows for the modelling of numerous three-dimensional physical systems (e.g., hydrogenic atoms and ions, exotic atoms, antimatter atoms, Rydberg atoms) and a number of nanotechnological objects (quantum wells, wires and dots) and qubits which have been shown to be very useful in semiconductor physics [43, 44] and quantum technologies [45, 46], respectively. Moreover, it plays a crucial role for the interpretation of numerous phenomena of quantum cosmology [47] and quantum field theory [48 50]. In addition, the $D$-dimensional hydrogenic wavefunctions have been used as complete orthonormal sets for many-body atomic and molecular problems [51, 52] in both position and momentum spaces. However, although tremendous advances have been witnessed in understanding the (energetic) spectroscopic properties of three-dimensional hydrogenic atoms, the informational properties of standard and non-standard hydrogenic systems are barely known. The latter properties, which crucially depend on the system's eigenfunctions, quantify the various facets of the spatial extension or multidimensional spreading of the electronic charge. The aim of this work is to cover this informational lack by means of the determination of the Rényi entropies for the multidimensional hydrogenic system.

The calculation of the hydrogenic Rényi entropies is a difficult task except for the lowest-lying energy states. This is because these quantities are described by means of some power or logarithmic functionals of the electron density, which cannot be easily handled in an analytical way nor numerically computed; the latter is basically because a naive numerical evaluation using quadratures is not convenient due to the increasing number of integrable singularities when the principal hyperquantum number $n$ is increasing, which spoils any attempt to achieve reasonable accuracy even for rather small $n$. Up until now, these quantities have been only calculated in a compact form [34, 35, 53] at the high-dimensional (pseudoclassical) and high-energy (Rydberg) limits by use of modern asymptotical techniques of the Laguerre and Gegenbauer polynomials 
which control the state's wavefunctions in position and momentum spaces [22, 54].

In this work we determine the Rényi entropies $R_{q}[\rho]$ (with integer $q$ greater than 1 ) for the electron density $\rho(\vec{r})$ of all the discrete stationary states of the $D$-dimensional hydrogenic system directly in terms of the hyperquantum numbers which characterize the states, the nuclear charge and the space dimensionality $D$. The structure of the manuscript is the following. In Sec. 2 the notion of the $q$ th-order Rényi entropy for a $D$-dimensional probability is given, and then the wavefunctions of the hydrogenic states in the $D$-dimensional configuration space are briefly described so as to express the associated probability densities. In Sec. 3 the position and momentum Rényi entropies are analytically determined by means of the little known polynomial linearization methodology of Srivastava-Niukkanen type [55-57]. In Sec. 4 the specific values for the entropies of some particularly relevant hydrogenic states are given to illustrate the applicability of our procedure. Finally, some concluding remarks and open problems are given.

\section{D-DIMENSIONAL HYDROGENIC SYSTEM: AN ENTROPIC VIEW}

In this section we briefly describe the quantum position and momentum probability setting of the $D$-dimensional hydrogenic system where the Rényi entropies are applied. For convenience we start with the definition of these entropies for a general multidimensional probability density, and then we give the known wavefunctions [36 39] of the system in both position and momentum spaces as well as the corresponding quantum probability densities.

\section{A. Rényi entropy}

The Rényi entropies $R_{q}[\rho]$ of a $D$-dimensional probability density $\rho(\vec{r})$ are defined as

$$
R_{q}[\rho]=\frac{1}{1-q} \ln W_{q}[\rho] ; \quad 0<q<\infty, \quad q \neq 1,
$$

where $W_{q}[\rho]$ denotes the entropic or Yule-Sichel frequency moment of order $q$ of $\rho(\vec{r})$ is given by

$$
W_{q}[\rho]=\int_{\mathbb{R}^{D}}[\rho(\vec{r})]^{q} d \vec{r}=\|\rho\|_{q}^{q} ; \quad q>0
$$

where the position $\vec{r}=\left(x_{1}, \ldots, x_{D}\right)$ is given in hyperspherical units as $\left(r, \theta_{1}, \theta_{2}, \ldots, \theta_{D-1}\right) \equiv$ $\left(r, \Omega_{D-1}\right), \Omega_{D-1} \in S^{D-1}$; and the volume element is

$$
d \vec{r}=r^{D-1} d r d \Omega_{D}, \quad d \Omega_{D-1}=\left(\prod_{j=1}^{D-2} \sin ^{2 \alpha_{j}} \theta_{j} d \theta_{j}\right) d \phi
$$


with $2 \alpha_{j}=D-j-1$. We have used $r \equiv|\vec{r}|=\sqrt{\sum_{i=1}^{D} x_{i}^{2}} \in[0 ;+\infty)$ and $x_{i}=r\left(\prod_{k=1}^{i-1} \sin \theta_{k}\right) \cos \theta_{i}$ for $1 \leq i \leq D$ and with $\theta_{i} \in[0 ; \pi), i<D-1, \theta_{D-1} \equiv \phi \in[0 ; 2 \pi)$. By convention $\theta_{D}=0$ and the empty product is the unity.

\section{B. Hydrogenic system}

The discrete stationary states of the $D$-dimensional hydrogenic system (i.e., a particle moving in the Coulomb potential $V_{D}(r)=-\frac{Z}{r}$, where $Z$ denotes the nuclear charge; atomic units are used throughout the paper) are known to be expressed [33, 37] in position space by the energy eigenvalues

$$
E=-\frac{Z^{2}}{2 \eta^{2}}, \quad \eta=n+\frac{D-3}{2} ; \quad n=1,2,3, \ldots,
$$

and the associated eigenfunctions

$$
\begin{aligned}
\Psi_{n, l,\{\mu\}}(\vec{r}) & =N_{n, l}\left(\frac{r}{\lambda}\right)^{l} e^{-\frac{r}{2 \lambda}} \mathcal{L}_{n-l-1}^{(2 l+D-2)}\left(\frac{r}{\lambda}\right) \mathcal{Y}_{l,\{\mu\}}\left(\Omega_{D-1}\right) \\
& =N_{\eta, l}\left[\frac{\omega_{2 L+1}(\tilde{r})}{\tilde{r}^{D-2}}\right]^{1 / 2} \mathcal{L}_{\eta-L-1}^{(2 L+1)}(\tilde{r}) \mathcal{Y}_{l,\{\mu\}}\left(\Omega_{D-1}\right)
\end{aligned}
$$

with

$$
\begin{aligned}
\eta & =n+\frac{D-3}{2}, \quad n=1,2,3, \ldots \\
L & =l+\frac{D-3}{2}, \quad l=0,1,2, \ldots \\
\tilde{r} & =\frac{r}{\lambda} \quad \text { with } \quad \lambda=\frac{\eta}{2 Z},
\end{aligned}
$$

The symbol $\eta$ denotes the principal hyperquantum number of the state associated to the radial coordinate, and $(l,\{\mu\}) \equiv\left(l \equiv \mu_{1}, \mu_{2}, \ldots, \mu_{D-1}\right)$ denote the orbital and magnetic hyperquantum numbers associated to the angular variables $\Omega_{D-1} \equiv\left(\theta_{1}, \theta_{2}, \ldots, \theta_{D-1}\right)$, which may take all values consistent with the inequalities $l \equiv \mu_{1} \geq \mu_{2} \geq \ldots \geq\left|\mu_{D-1}\right| \equiv|m| \geq 0$. In addition, $\omega_{\alpha}(x)=x^{\alpha} e^{-x}, \alpha=2 l+D-2$ is the weight function of the orthogonal and orthonormal Laguerre polynomials [58, 59] of degree $n$ and parameter $\alpha$, here denoted by $L_{n}^{(\alpha)}(x)$ and $\widehat{L}_{n}^{(\alpha)}(x)$, respectively and

$$
N_{n, l}=\lambda^{-\frac{D}{2}}\left(\frac{(\eta-L-1) !}{2 \eta(\eta+L) !}\right)^{\frac{1}{2}}
$$

is the normalization constant which ensures the unit norm of the wavefunction. The angular part of the eigenfunctions is given by the hyperspherical harmonics as

$$
\mathcal{Y}_{l,\{\mu\}}\left(\Omega_{D-1}\right)=\mathcal{N}_{l,\{\mu\}} e^{i m \phi} \times \prod_{j=1}^{D-2} \mathcal{C}_{\mu_{j}-\mu_{j+1}}^{\left(\alpha_{j}+\mu_{j+1}\right)}\left(\cos \theta_{j}\right)\left(\sin \theta_{j}\right)^{\mu_{j+1}}
$$


where $\mathcal{N}_{l,\{\mu\}}$ is the normalization constant

$$
\mathcal{N}_{l,\{\mu\}}^{2}=\frac{1}{2 \pi} \times \prod_{j=1}^{D-2} \frac{\left(\alpha_{j}+\mu_{j}\right)\left(\mu_{j}-\mu_{j+1}\right) !\left[\Gamma\left(\alpha_{j}+\mu_{j+1}\right)\right]^{2}}{\pi 2^{1-2 \alpha_{j}-2 \mu_{j+1}} \Gamma\left(2 \alpha_{j}+\mu_{j}+\mu_{j+1}\right)}
$$

the symbol $\mathcal{C}_{n}^{(\lambda)}(t)$ denotes the Gegenbauer polynomial [58, 59] of degree $n$ and parameter $\lambda$, and $2 \alpha_{j}=D-j-1$.

Then, the position probability density of a $D$-dimensional hydrogenic state characterized by the hyperquantum numbers $(n, l,\{\mu\})$ is given by the squared modulus of the position eigenfunction as

$$
\begin{aligned}
\rho_{n, l,\{\mu\}}(\vec{r}) & =N_{\eta, l}^{2}\left[\frac{\omega_{2 L+1}(\tilde{r})}{\tilde{r} D-2}\right]\left[\mathcal{L}_{\eta-L-1}^{(2 L+1)}(\tilde{r})\right]^{2}\left|\mathcal{Y}_{l,\{\mu\}}\left(\Omega_{D-1}\right)\right|^{2} \\
& =N_{n, l}^{2} \tilde{r}^{2 l} e^{-\tilde{r}}\left[\mathcal{L}_{n-l-1}^{(2 l+D-2)}(\tilde{r})\right]^{2}\left|\mathcal{Y}_{l,\{\mu\}}\left(\Omega_{D-1}\right)\right|^{2} \\
& \equiv \rho_{n, l}(\tilde{r})\left|\mathcal{Y}_{l,\{\mu\}}\left(\Omega_{D-1}\right)\right|^{2}
\end{aligned}
$$

Moreover, the Fourier transform of the position eigenfunction $\Psi_{\eta, l,\{\mu\}}(\vec{r})$ given by (6) , i.e., $\tilde{\Psi}(\vec{p})=\int_{\mathbb{R}^{D}} e^{-i \vec{p} \cdot \vec{r}} \Psi(\vec{r}) \frac{d \vec{r}}{(2 \pi)^{\frac{D}{2}}}$, provides the eigenfunction of the system in the momentum space as

$$
\tilde{\Psi}_{n, l,\{\mu\}}(\vec{p})=\mathcal{M}_{n, l}(p) \mathcal{Y}_{l,\{\mu\}}\left(\Omega_{D-1}\right),
$$

where the radial part is

$$
\mathcal{M}_{n, l}(p)=K_{n, l} \frac{(\eta \tilde{p})^{l}}{\left(1+\eta^{2} \tilde{p}^{2}\right)^{L+2}} \mathcal{C}_{\eta-L-1}^{(L+1)}\left(\frac{1-\eta^{2} \tilde{p}^{2}}{1+\eta^{2} \tilde{p}^{2}}\right)
$$

with $\tilde{p}=\frac{p}{Z}$ and the normalization constant

$$
K_{n, l}=Z^{-\frac{D}{2}} 2^{2 L+3}\left[\frac{(\eta-L-1) !}{2 \pi(\eta+L) !}\right]^{\frac{1}{2}} \Gamma(L+1) \eta^{\frac{D+1}{2}} .
$$

Then, the momentum probability density of the $D$-dimensional hydrogenic stationary state with the hyperquantum numbers $(n, l,\{\mu\})$ is

$$
\begin{aligned}
& \gamma_{n, l,\{\mu\}}(\vec{p})=\left|\tilde{\Psi}_{n, l,\{\mu\}}(\vec{p})\right|^{2}=\mathcal{M}_{n, l}^{2}(p)\left|\mathcal{Y}_{l,\{\mu\}}\left(\Omega_{D-1}\right)\right|^{2} \\
& \quad=K_{n, l}^{2} \frac{(\eta \tilde{p})^{2 l}}{\left(1+\eta^{2} \tilde{p}^{2}\right)^{2 L+4}}\left[\mathcal{C}_{\eta-L-1}^{(L+1)}\left(\frac{1-\eta^{2} \tilde{p}^{2}}{1+\eta^{2} \tilde{p}^{2}}\right)\right]^{2}\left|\mathcal{Y}_{l,\{\mu\}}\left(\Omega_{D-1}\right)\right|^{2}
\end{aligned}
$$

Note that the position and momentum probability densities are quite different since the radial parts of the densities are controlled by the Laguerre and the Gegenbauer polynomials in the position and momentum cases, respectively. Then, we expect the Rényi entropies of the hydrogenic 
system in the position and momentum spaces to be different; this is not surprising because the Rényi entropies are not observables of the system. They satisfy the known Rényi-entropy-based uncertainty relation [25, 61 63] as given by Eq. (44) below. The latter relation indicates that the joint position-momentum Rényi entropy, which is given by the sum of the position and momentum Rényi entropies, is bounded from below for all bound stationary states of the system.

\section{EXACT RÉNYI ENTROPIES OF THE HYDROGENIC SYSTEM}

In this section we determine the position and momentum Rényi entropies $R_{q}[\rho]$ (with natural $q$ other than unity) for all the discrete stationary states of the $D$-dimensional hydrogenic system in an analytical way. First we note that these entropies can be decomposed into two radial and angular parts in both conjugated spaces. Then, we use a recent procedure [18] based on the Srivastava-Niukkanen method [55, 56, 60] which linearize integer powers of Laguerre and Jacobi polynomials. The involved linearization coefficients are expressed via some multiparametric hypergeometric functions of Lauricella and Srivastava-Daoust types, respectively.

From Eqs. (2), (3) and (10) the Rényi entropies of the $D$-dimensional hydrogenic state $(n, l,\{\mu\})$ in position space can be written as

$$
R_{q}\left[\rho_{n, l,\{\mu\}}\right]=R_{q}\left[\rho_{n, l}\right]+R_{q}\left[\mathcal{Y}_{l,\{\mu\}}\right]
$$

where $R_{q}\left[\rho_{n, l}\right]$ denotes the radial part

$$
R_{q}\left[\rho_{n, l}\right]=\frac{1}{1-q} \ln \int_{0}^{\infty}\left[\rho_{n, l}\right]^{q} r^{D-1} d r
$$

and $R_{q}\left[\mathcal{Y}_{l,\{\mu\}}\right]$ denotes the angular part

$$
R_{q}\left[\mathcal{Y}_{l,\{\mu\}}\right]=\frac{1}{1-q} \ln \Lambda_{l,\{\mu\}}(q),
$$

with

$$
\Lambda_{l,\{\mu\}}(q)=\int\left|\mathcal{Y}_{l,\{\mu\}}\left(\Omega_{D-1}\right)\right|^{2 q} d \Omega_{D-1}
$$




\section{A. Radial Rényi entropy in position space}

Taking into account Eqs. (10) and (16), the radial Rényi entropy can be written as

$$
\begin{aligned}
R_{q}\left[\rho_{n, l}\right] & =\frac{1}{1-q} \ln \left[N_{n, l}^{2 q} \int_{0}^{\infty} \tilde{r}^{2 l q} e^{-q \tilde{r}}\left[\mathcal{L}_{n-l-1}^{(2 l+D-2)}(\tilde{r})\right]^{2 q} r^{D-1} d r\right], \\
& =\frac{1}{1-q} \ln \left[\lambda^{D(1-q)}\left(\frac{\Gamma(n-l)}{2 \eta \Gamma(n+l+D-2)}\right)^{q}\right] \\
& +\frac{1}{1-q} \ln \int_{0}^{\infty} \tilde{r}^{2 l q+D-1} e^{-q \tilde{r}}\left[\mathcal{L}_{n-l-1}^{(2 l+D-2)}(\tilde{r})\right]^{2 q} d \tilde{r}
\end{aligned}
$$

To evaluate the integral first we perform the change of variable $x=q \tilde{r}$ to have

$$
\begin{aligned}
& R_{q}\left[\rho_{n, l}\right]=\frac{1}{1-q} \ln \left[\lambda^{D(1-q)}\left(\frac{\Gamma(n-l)}{2 \eta \Gamma(n+l+D-2)}\right)^{q}\right] \\
& +\frac{1}{1-q} \ln q^{-D-2 l q} \int_{0}^{\infty} x^{2 l q+D-1} e^{-x}\left[\mathcal{L}_{n-l-1}^{(2 l+D-2)}\left(\frac{x}{q}\right)\right]^{2 q} d x
\end{aligned}
$$

and then we apply the linearization formula of the $(2 q)$ th-power of the Laguerre polynomial $L_{n-l-1}^{(2 l+D-2)}\left(\frac{x}{q}\right)$ given by

$$
y^{a}\left[\mathcal{L}_{k}^{(\alpha)}(t y)\right]^{r}=\sum_{i=0}^{\infty} c_{i}(a, r, t, k, \alpha, \gamma) \mathcal{L}_{i}^{(\gamma)}(y),
$$

with $a>0, t>0, \alpha>-1, \gamma>-1$, the integer $k \geq 0, i \geq 0$, and the linearization coefficients

$$
\left.\begin{array}{rl}
c_{i}(a, r, t, k, \alpha, \gamma)= & (\gamma+1)_{a}\left(\frac{\Gamma(k+\alpha+1)}{\Gamma(\alpha+1) \Gamma(k+1)}\right)^{r} \\
\times F_{A}^{(r+1)} & (\begin{array}{c}
\gamma+a+1 ; \overbrace{-k, \ldots,-k},-i \\
\underbrace{\alpha+1, \ldots, \alpha+1}_{r}, \gamma+1
\end{array} ; \underbrace{t, \ldots, t}_{r}, 1
\end{array}\right),
$$

where the Pochhammer symbol $(z)_{a}=\frac{\Gamma(z+a)}{\Gamma(z)}$ and the symbol $F_{A}^{(s)}\left(x_{1}, \ldots, x_{r}\right)$ denotes the Lauricella function of type A of $s$ variables and $2 s+1$ parameters defined as [60]

$$
F_{A}^{(s)}\left(\begin{array}{c}
a ; b_{1}, \ldots, b_{s} \\
\\
c_{1}, \ldots, c_{s}
\end{array} x_{1}, \ldots, x_{s}\right)=\sum_{j_{1}, \ldots, j_{s}=0}^{\infty} \frac{(a)_{j_{1}+\ldots+j_{s}}\left(b_{1}\right)_{j_{1}} \cdots\left(b_{s}\right)_{j_{s}}}{\left(c_{1}\right)_{j_{1}} \cdots\left(c_{s}\right)_{j_{s}}} \frac{x_{1}^{j_{1}} \cdots x_{s}^{j_{s}}}{j_{1} ! \cdots j_{s} !} .
$$

Now, taking $a=2 l q+D-1, r=2 q, t=\frac{1}{q}, k=n-l-1, \alpha=2 l+D-2$, inserting (21) in the integral kernel of (20) and using the orthogonalization condition of the Laguerre polynomials [59], 
after some algebraic manipulations one finds that the final expression of the radial Rényi entropy is given by

$R_{q}\left[\rho_{n, l}\right]=D \ln \left(\frac{\eta}{2 Z}\right)+\frac{q}{1-q} \ln \left(\frac{(\eta-L)_{2 L+1}}{2 \eta}\right)+\frac{1}{1-q} \ln \mathcal{F}_{q}(D, \eta, L)+\frac{1}{1-q} \ln \mathcal{A}_{q}(D, L)$

where

$$
\mathcal{F}_{q}(D, n, l) \equiv F_{A}^{(2 q)}\left(\begin{array}{cc}
2 l q+D ; \overbrace{-n+l+1, \ldots,-n+l+1}^{2 q} & ; \underbrace{\frac{1}{q}, \ldots, \frac{1}{q}}_{2 q} \\
\underbrace{2 l+D-1, \ldots, 2 l+D-1}_{2 q}
\end{array}\right),
$$

and $\mathcal{A}_{q}(D, L) \equiv \frac{\Gamma(D+2 l q)}{q^{D+2 l q} \Gamma(2 L+2)^{2 q}}$. Note that when $l=n-1$ the function $\mathcal{F}_{q}(D, n, l)$ is equal to unity, so that the third term of the entropy expression (24) vanishes. Moreover, let us highlight that, from Eq. (23), this function defines a finite sum by taking into account the properties of the involved Pochhammer symbols with negative integer arguments.

\section{B. Angular Rényi entropy}

Now, from Eqs. (2), (3), (17), (18) and (9) one has that the angular Rényi entropy has the form

$$
R_{q}\left[\mathcal{Y}_{l,\{\mu\}}\right]=\frac{1}{1-q} \ln \int \mathcal{N}_{l,\{\mu\}}^{2 q} \prod_{j=1}^{D-2}\left[\mathcal{C}_{\mu_{j}-\mu_{j+1}}^{\left(\alpha_{j}+\mu_{j+1}\right)}\left(\cos \theta_{j}\right)\right]^{2 q}\left|\sin \theta_{j}\right|^{2 q \mu_{j+1}} d \Omega_{D-1}
$$

With the change of variable $t=\cos \theta_{j}$, this integral can be rewritten as

$$
R_{q}\left[\mathcal{Y}_{l,\{\mu\}}\right]=\frac{1}{1-q} \ln \left(2 \pi \mathcal{N}_{l,\{\mu\}}^{2 q}\right)+\frac{1}{1-q} \ln \left[\prod_{j=1}^{D-2} \mathcal{I}_{j}(q)\right]
$$

where

$$
\mathcal{I}_{q}\left(D, \mu_{j}, \mu_{j+1}\right)=\int_{-1}^{1}\left[\mathcal{C}_{\mu_{j}-\mu_{j+1}}^{\left(\alpha_{j}+\mu_{j+1}\right)}(t)\right]^{2 q}\left|\left(1-t^{2}\right)\right|^{q \mu_{j+1}+\alpha_{j}-\frac{1}{2}} d t
$$

To calculate this integral we use the known relationship between the Gegenbauer and Jacobi polynomials [59],

$$
\mathcal{C}_{\kappa}^{(\lambda)}(x)=\frac{\Gamma\left(\lambda+\frac{1}{2}\right)}{\Gamma(2 \lambda)} \frac{\Gamma(\kappa+2 \lambda)}{\Gamma\left(\kappa+\lambda+\frac{1}{2}\right)} \mathcal{P}_{\kappa}^{\left(\lambda-\frac{1}{2}, \lambda-\frac{1}{2}\right)}(x)
$$

together with the Srivastava-Niukkanen-based linearization formula of the Jacobi polynomials [18]

$$
\left[\mathcal{P}_{\kappa}^{(\alpha, \beta)}(x)\right]^{2 q}=\sum_{i=0}^{\infty} \tilde{c}_{i}(0,2 q, \kappa, \alpha, \beta, \gamma, \delta) \mathcal{P}_{i}^{(\gamma, \delta)}(x)
$$


with $\alpha>-1, \beta>-1, \gamma>-1, \delta>-1$ and where the linearization coefficients $\tilde{c}_{i}$ are given by

$$
\begin{aligned}
& \tilde{c}_{i}(0,2 q, \kappa, \alpha, \beta, \gamma, \delta)=\left(\frac{\Gamma(\kappa+\alpha+1)}{\Gamma(\alpha+1) \Gamma(\kappa+1)}\right)^{2 q} \frac{\gamma+\delta+2 i+1}{\gamma+\delta+i+1} \\
& \times \sum_{\substack{j_{1}, \ldots, j_{2 q}=0 \\
j_{2 q+1}=0}}^{i} \frac{(\gamma+1)_{j_{1}+\ldots+j_{2 q}+j_{2 q+1}}}{(\gamma+\delta+i+2)_{j_{1}+\ldots+j_{2 q}}} \\
& \times \frac{(-\kappa)_{j_{1}}(\alpha+\beta+\kappa+1)_{j_{1}} \cdots(-\kappa)_{j_{2 q}}(\alpha+\beta+\kappa+1)_{j_{2 q}}(-i)_{j_{2 q+1}}}{(\alpha+1)_{j_{1}} \cdots(\alpha+1)_{j_{2 q}}(\gamma+1)_{j_{2 q+1}} j_{1} ! \cdots j_{2 q} ! j_{2 q+1} !} .
\end{aligned}
$$

Then, the orthogonalization relation of the Jacobi polynomials [59]

$\int_{-1}^{1}(1-x)^{\alpha}(1+x)^{\beta} P_{m}^{(\alpha, \beta)}(x) P_{n}^{(\alpha, \beta)}(x) d x=\frac{2^{\alpha+\beta+1}}{n !} \frac{\Gamma(\alpha+n+1) \Gamma(\beta+n+1)}{(\alpha+\beta+2 n+1) \Gamma(\alpha+\beta+n+1)} \delta_{m, n}$,

(for $\alpha, \beta>-1$, and where $\delta_{n, m}$ denotes the Kronecker's delta function) reduces the infinite number of infinite terms of the sum involved in our Gegenbauer linearization to a single one: that for $i=0$. Then, after some algebraic manipulations one obtains that the analytical expression of the angular Rényi entropy is given by

$$
\begin{gathered}
R_{q}\left[\mathcal{Y}_{l,\{\mu\}}\right]=\ln \left(2 \pi^{\frac{D}{2}}\right)+\frac{1}{1-q} \ln \left[\frac{\Gamma\left(l+\frac{D}{2}\right)^{q}}{\Gamma\left(q l+\frac{D}{2}\right)} \frac{\Gamma(q m+1)}{\Gamma(m+1)^{q}}\right] \\
+\frac{1}{1-q} \sum_{j=1}^{D-2} \ln \left[\mathcal{B}_{q}\left(D, \mu_{j}, \mu_{j+1}\right) \mathcal{G}_{q}\left(D, \mu_{j}, \mu_{j+1}\right)\right]
\end{gathered}
$$

where

$\mathcal{B}_{q}\left(D, \mu_{j}, \mu_{j+1}\right)=\frac{1}{\left[\left(\mu_{j}-\mu_{j+1}\right) !\right]^{q}} \frac{\left(2 \alpha_{j}+2 \mu_{j+1}+1\right)_{2\left(\mu_{j}-\mu_{j+1}\right)}^{q}}{\left(2 \alpha_{j}+\mu_{j}+\mu_{j+1}\right)_{\mu_{j}-\mu_{j+1}}^{q}} \frac{\left(q \mu_{j+1}+\alpha_{j}+1\right)_{q\left(\mu_{j}-\mu_{j+1}\right)}}{\left(\alpha_{j}+\mu_{j+1}+1\right)_{\mu_{j}-\mu_{j+1}}^{q}}$

and

$$
\begin{aligned}
\mathcal{G}_{q}\left(D, \mu_{j}, \mu_{j+1}\right) & =F_{1: 1 ; \ldots ; 1}^{1: 2 ; \ldots ; 2}\left(\begin{array}{c}
a_{j}: b_{j}, c_{j} ; \ldots ; b_{j}, c_{j} \\
d_{j}: e_{j} ; \ldots ; e_{j}
\end{array}\right) \\
& =\sum_{i_{1}, \ldots, i_{2 q}=0}^{\mu_{j}-\mu_{j+1}} \frac{\left(a_{j}\right)_{i_{1}+\ldots i_{2 q}}}{\left(d_{j}\right)_{i_{1}+\ldots+i_{2 q}}} \frac{\left(b_{j}\right)_{i_{1}}\left(c_{j}\right)_{i_{1}} \cdots\left(b_{j}\right)_{i_{2 q}}\left(c_{j}\right)_{i_{2 q}}}{\left(e_{j}\right)_{i_{1}} \cdots\left(e_{j}\right)_{i_{2 q}} i_{1} ! \cdots i_{2 q} !}
\end{aligned}
$$

with $a_{j}=\alpha_{j}+q \mu_{j+1}+\frac{1}{2}, b_{j}=-\mu_{j}+\mu_{j+1}, c_{j}=2 \alpha_{j}+\mu_{j+1}+\mu_{j}, d_{j}=2 q \mu_{j+1}+2 \alpha_{j}+1$ and $e_{j}=\alpha_{j}+\mu_{j+1}+\frac{1}{2}$. Note that the sum becomes finite because $b_{j}$ is a negative integer number, and so $\left(b_{j}\right)_{i}=\frac{\Gamma\left(b_{j}+i\right)}{\Gamma\left(b_{j}\right)}=0, \quad \forall i>\left|b_{j}\right|$. Let us also highlight that when $\mu_{j}=\mu_{j+1}$, the function $\mathcal{B}_{q}\left(D, \mu_{j}, \mu_{j+1}\right)=\mathcal{G}_{q}\left(D, \mu_{j}, \mu_{j+1}\right)=1$. The symbol $F_{1: 1 ; \ldots ; 1}^{1: 2 ; \ldots ; 2}\left(x_{1}, \ldots, x_{r}\right)$ denotes the 
$r$-variate Srivastava-Daoust function [18, 55] defined as

$$
\begin{array}{r}
F_{1: 1 ; \ldots ; 1}^{1: 2 ; \ldots ; 2}\left(\begin{array}{c}
a_{0}^{(1)}: a_{1}^{(1)}, a_{1}^{(2)} ; \ldots ; a_{r}^{(1)}, a_{r}^{(2)} \\
b_{0}^{(1)}: b_{1}^{(1)} ; \ldots ; b_{r}^{(1)}
\end{array} ; x_{1}, \ldots, x_{r}\right)= \\
=\sum_{j_{1}, \ldots, j_{r}=0}^{\infty} \frac{\left(a_{0}^{(1)}\right)_{j_{1}+\ldots+j_{r}} \frac{\left(a_{1}^{(1)}\right)_{j_{1}}\left(a_{1}^{(2)}\right)_{j_{1}} \ldots\left(a_{r}^{(1)}\right)_{j_{r}}\left(a_{r}^{(2)}\right)_{j_{r}}}{\left(b_{0}^{(1)}\right)_{j_{1}+\ldots+j_{r}}} \frac{x_{1}^{j_{1}} x_{2}^{j_{2}} \cdots x_{r}^{j_{r}}}{j_{1} ! j_{2} ! \cdots j_{r} !}}{\left(b_{r}^{(1)}\right)_{j_{r}}}
\end{array}
$$

Let us highlight that Eqs. (32)-(35) give the angular contribution to the Rényi entropies not only for the hydrogenic systems, but also for any non-relativistic and spherically-symmetric multidimensional quantum system. Note from Eq. (32) that the first term depends only on the spatial dimensionality and the second term on the first and last hyperquantum angular numbers, the spatial dimensionality and the entropic parameter $q$. Finally, the last term represents the sum of the different contributions of the internal angular degrees of freedom $\left\{\mu_{j}\right\}_{j=2}^{D-2}$, which depends on the corresponding hyperquantum numbers $\mu_{j}$ and $\mu_{j+1}$.

\section{Total Rényi entropy in position space}

Finally, from Eqs. (15), (24) and (32) one has that the total Rényi entropy of the $D$-dimensional hydrogenic system in position space is given by

$$
\begin{aligned}
R_{q}\left[\rho_{n, l,\{\mu\}}\right]= & D \ln \left(\frac{\pi^{\frac{1}{2}} \eta}{2 Z}\right)+\frac{q}{1-q} \ln \left(\frac{(\eta-L)_{2 L+1}}{2 \eta}\right) \\
& +\frac{1}{1-q} \ln \mathcal{F}_{q}(D, \eta, L) \mathcal{A}_{q}(D, L)+\frac{1}{1-q} \ln \left[\frac{\Gamma\left(l+\frac{D}{2}\right)^{q}}{\Gamma\left(q l+\frac{D}{2}\right)} \frac{\Gamma(q m+1)}{\Gamma(m+1)^{q}}\right] \\
& +\frac{1}{1-q} \sum_{j=1}^{D-2} \ln \left[\mathcal{B}_{q}\left(D, \mu_{j}, \mu_{j+1}\right) \mathcal{G}_{q}\left(D, \mu_{j}, \mu_{j+1}\right)\right]+\ln 2
\end{aligned}
$$

in terms of the hyperquantum numbers, the nuclear charge and the space dimensionality. 


\section{Radial and total Rényi entropy in momentum space}

Operating in momentum space in a similar way as done for the position space in subsection 3.1, one has from Eqs. (2), (3) and (14) that the momentum radial Rényi entropy is given by

$$
\begin{aligned}
R_{q}\left[\gamma_{n, l}\right]= & \frac{1}{1-q} \ln \left(\frac{Z^{D}}{\eta^{D}} \frac{K_{n, l}^{2 q}}{2^{q(L+2)}}\right) \\
& +\frac{1}{1-q} \ln \int_{-1}^{1}(1-y)^{l q+\frac{D}{2}-1}(1+y)^{D\left(q-\frac{1}{2}\right)+q(l+1)-1} \mathcal{C}_{n-l-1}^{(L+1)}(y)^{2 q} d y
\end{aligned}
$$

Again the use of the relation (29) and the Srivastava-Niukkanen-based linearization formula (30) of the Jacobi polynomials has led us to find the following expression of the radial part of the Rényi entropy in momentum space:

$$
\begin{aligned}
R_{q}\left[\gamma_{n, l}\right] & =D \ln \frac{Z}{\eta}+\frac{q}{1-q} \ln \left[2 \eta(\eta-L)_{2 L+1}\right] \\
& +\frac{1}{1-q} \ln \overline{\mathcal{F}}_{q}(D, \eta, L)+\frac{1}{1-q} \ln \overline{\mathcal{A}}_{q}(D, L)
\end{aligned}
$$

where

$$
\begin{gathered}
\overline{\mathcal{F}}_{q}(D, \eta, L) \equiv F_{1: 1 ; \ldots ; 1}^{1: 2 ; \ldots ; 2}\left(\begin{array}{c}
a: b, c ; \ldots ; b, c \\
d: e ; \ldots ; e
\end{array}\right) \\
=\sum_{i_{1}, \ldots, i_{2 q}=0}^{n-l-1} \frac{(a)_{i_{1}+\ldots i_{2 q}}}{(d)_{i_{1}+\ldots+i_{2 q}}} \frac{(b)_{i_{1}}(c)_{i_{1}} \cdots(b)_{i_{2 q}}(c)_{i_{2 q}}}{(e)_{i_{1}} \cdots(e)_{i_{2 q}} i_{1} ! \cdots i_{2 q} !}
\end{gathered}
$$

with $a=\left(L+\frac{3}{2}\right) q+\frac{D}{2}(1-q), b=-(\eta-L-1), c=\eta+L+1, d=q(2 L+4), e=L+\frac{3}{2}$ and

$$
\overline{\mathcal{A}}_{q}(D, L) \equiv 2^{2 q-1} \frac{\Gamma\left(\frac{D}{2}+q l\right) \Gamma\left(-\frac{D}{2}+q(D+l+1)\right)}{\Gamma\left(\frac{D}{2}+l\right)^{2 q} \Gamma(q(D+2 l+1))}
$$

Note that, when $l=n-1$ the function $\mathcal{F}_{q}(D, \eta, L)=1$.

Finally, since the angular part of the momentum Rényi entropy is the same as in position space, one obtains from Eqs. (38) and (32) that the total Rényi entropy in momentum space $R_{q}\left[\gamma_{\eta, L,\left\{\mu_{j}\right\}}\right]=R_{q}\left[\gamma_{n, l}\right]+R_{q}\left[\mathcal{Y}_{l,\{\mu\}}\right]$ has the following expression

$$
\begin{aligned}
& R_{q}\left[\gamma_{n, l,\{\mu\}}\right]=D \ln \left(\frac{\pi^{\frac{1}{2}} Z}{\eta}\right)+\frac{q}{1-q} \ln \left[2 \eta(\eta-L)_{2 L+1}\right] \\
& \quad+\frac{1}{1-q} \ln \left[\overline{\mathcal{F}}_{q}(D, \eta, L) \overline{\mathcal{A}}_{q}(D, L) \frac{\Gamma\left(l+\frac{D}{2}\right)^{q}}{\Gamma\left(q l+\frac{D}{2}\right)} \frac{\Gamma(q m+1)}{\Gamma(m+1)^{q}}\right] \\
& +\frac{1}{1-q} \sum_{j=1}^{D-2} \ln \left[\mathcal{B}_{q}\left(D, \mu_{j}, \mu_{j+1}\right) \mathcal{G}_{q}\left(D, \mu_{j}, \mu_{j+1}\right)\right]+\ln 2
\end{aligned}
$$

in terms of the hyperquantum numbers, the nuclear charge and the space dimensionality. 


\section{E. Rényi entropies for the quasi-spherical $\mathrm{n} s$ states}

To illustrate the applicability of the previous position and momentum Rényi entropies, we calculate them for a relevant class of specific states of the $D$-dimensional hydrogenic system which include the ground state: the quasi-spherical n $s$ states, which are characterized by the angular hyperquantum numbers $\mu_{1}=\mu_{2} \ldots=\mu_{D-1}=l$. First, since $l=n-1$, the Lauricella function of Eq. (24) is equal to unity. Then, we find the values

$$
R_{q}\left[\rho_{n, n-1}\right]=D \ln \frac{\eta}{2 Z}-\frac{q}{1-q} \ln [\Gamma(2 \eta+1)]+\frac{1}{1-q} \ln \left(\frac{\Gamma(D+2 n q-2 q)}{q^{D+2 n q-2 q}}\right)
$$

for the radial Rényi entropy of the $\mathrm{n} s$ states in position space, and

$$
R_{q}\left[\rho_{1,0}\right]=\Gamma(D)+D \ln \left[\frac{D-1}{4 Z q^{\frac{1}{1-q}}}\right]
$$

for the corresponding one of the ground state $(n=1)$. In addition, we have found the values

$$
R_{q}\left[\mathcal{Y}_{l,\{\mu\}}\right]=\ln \left(2 \pi^{\frac{D}{2}}\right)+\frac{1}{1-q} \ln \left[\frac{\Gamma\left(l+\frac{D}{2}\right)^{q}}{\Gamma(l+1)^{q}} \frac{\Gamma(q l+1)}{\Gamma\left(q l+\frac{D}{2}\right)}\right]
$$

and

$$
R_{q}\left[\mathcal{Y}_{0,\{0\}}\right]=\ln \left[\frac{2 \pi^{\frac{D}{2}}}{\Gamma\left(\frac{D}{2}\right)}\right]
$$

for the angular Rényi entropy of the n $s$ states and the ground state, respectively. Similar operations in the momentum space have allowed us to have the values

$$
\begin{aligned}
& R_{q}\left[\gamma_{n, n-1}\right]=D \ln \frac{Z}{\eta}+\frac{q}{1-q} \ln [4 \Gamma(2 \eta+1)] \\
&+\frac{1}{1-q} \ln \left[\frac{\Gamma\left(\frac{D}{2}+q n-q\right) \Gamma\left(-\frac{D}{2}+q(D+n)\right)}{2 \Gamma\left(n+\frac{D}{2}-1\right)^{2 q} \Gamma(q(D+2 n-1))}\right]
\end{aligned}
$$

and

$$
\begin{aligned}
R_{q}\left[\gamma_{1,0}\right] & =D \ln \left[\frac{2 Z}{D-1}\right]+\frac{q}{1-q} \ln [4 \Gamma(D)] \\
& +\frac{1}{1-q} \ln \left[\frac{\Gamma\left(\frac{D}{2}\right)^{1-2 q} \Gamma\left(D\left(q-\frac{1}{2}\right)+q\right)}{2 \Gamma(D q+q)}\right]
\end{aligned}
$$

for for the radial Rényi entropy of the $\mathrm{n} s$ states and the ground state in momentum space, respectively. 
Finally, we gather in Tables 1 and 2 the exact values of the position and momentum Rényi entropies $R_{2}\left[\rho_{n, l, m}\right]$ and $R_{2}\left[\gamma_{n, l, m}\right]$, respectively, of various quasi-circular circular states of the threedimensional hydrogen atom. Therein, we observe that this quantity increases (decreases) when the main quantum number $n$ is increasing (decreasing) in position space, and the opposite behavior is observed in momentum space. Moreover, it decreases (increases) when the orbital quantum number $l$ is increasing (decreasing) in position space, and the opposite behavior is observed in momentum space. And, the associated joint position-momentum entropy sum increases in a systematic way when $n$ or $l$ are increasing.

\begin{tabular}{|c|c|c|c|}
\hline$R_{2}\left[\rho_{n, l, m}\right]$ & $n=1$ & $n=2$ & $n=3$ \\
\hline$l=0, m=0$ & $\ln (8 \pi)$ & $\ln \left(\frac{2048 \pi}{5}\right)$ & $\ln \left(\frac{20736 \pi}{5}\right)$ \\
\hline$l=1, m=0$ & - & $\ln \left(\frac{2048 \pi}{9}\right)$ & $\ln \left(\frac{27648 \pi}{11}\right)$ \\
\hline$l=1, m=1$ & - & $\ln \left(\frac{1024 \pi}{3}\right)$ & $\ln \left(\frac{41472 \pi}{11}\right)$ \\
\hline$l=2, m=0$ & - & - & $\ln \left(\frac{9216 \pi}{5}\right)$ \\
\hline$l=2, m=1$ & - & - & $\ln \left(\frac{13824 \pi}{5}\right)$ \\
\hline$l=2, m=2$ & - & - & $\ln \left(\frac{13824 \pi}{5}\right)$ \\
\hline
\end{tabular}

TABLE I: Exact values of the total position Rényi entropy $R_{2}\left[\rho_{n, l, m}\right]$ for various quasi-circular states of the three-dimensional hydrogen atom. 


\begin{tabular}{|c|c|c|c|}
\hline$R_{2}\left[\gamma_{n, l, m}\right]$ & $n=1$ & $n=2$ & $n=3$ \\
\hline$l=0, m=0$ & $\ln \left(\frac{16 \pi^{2}}{33}\right)$ & $\ln \left(\frac{2 \pi^{2}}{151}\right)$ & $\ln \left(\frac{16 \pi^{2}}{7533}\right)$ \\
\hline$l=1, m=0$ & - & $\ln \left(\frac{2 \pi^{2}}{39}\right)$ & $\ln \left(\frac{160 \pi^{2}}{36207}\right)$ \\
\hline$l=1, m=1$ & - & $\ln \left(\frac{\pi^{2}}{13}\right)$ & $\ln \left(\frac{80 \pi^{2}}{12069}\right)$ \\
\hline$l=2, m=0$ & - & - & $\ln \left(\frac{1120 \pi^{2}}{78489}\right)$ \\
\hline$l=2, m=1$ & - & - & $\ln \left(\frac{560 \pi^{2}}{26163}\right)$ \\
\hline$l=2, m=2$ & - & - & $\ln \left(\frac{560 \pi^{2}}{26163}\right)$ \\
\hline
\end{tabular}

TABLE II: Exact values of the total momentum Rényi entropy $R_{2}\left[\gamma_{n, l, m}\right]$ for various quasi-circular states of the three-dimensional hydrogen atom.

\section{POSITION-MOMENTUM RÉNYI-ENTROPY SUM}

Here we give the joint position-momentum Rényi uncertainty sum for all the discrete stationary states of the $D$-dimensional hydrogenic system from Eqs. (36) and (40). We obtain

$$
\begin{aligned}
& R_{q}\left[\rho_{n, l,\{\mu\}}\right]+R_{p}\left[\gamma_{n, l,\{\mu\}}\right]=D \ln \left(\frac{\pi}{2}\right)+\frac{2 q}{q-1} \ln [2 \eta] \\
+ & \ln \left[\mathcal{F}_{q}(D, \eta, L)^{\frac{1}{1-q}} \overline{\mathcal{F}}_{p}(D, \eta, L)^{\frac{1}{1-p}} \mathcal{A}_{q}(D, L)^{\frac{1}{1-q}} \overline{\mathcal{A}}_{p}(D, L)^{\frac{1}{1-p}}\right] \\
+ & \ln \left[\left(\frac{\Gamma(q m+1)}{\Gamma\left(q l+\frac{D}{2}\right)}\right)^{\frac{1}{1-q}}\left(\frac{\Gamma(p m+1)}{\Gamma\left(p l+\frac{D}{2}\right)}\right)^{\frac{1}{1-p}}\right] \\
+ & \sum_{j=1}^{D-2} \ln \left[\left(q \mu_{j+1}+\alpha_{j}+1\right)_{q\left(\mu_{j+1}-\mu_{j}\right)}^{\frac{1}{1-q}}\left(p \mu_{j+1}+\alpha_{j}+1\right)^{\frac{1}{1-p}}\left(\mu_{j+1}-\mu_{j}\right)\right] \\
& +\sum_{j=1}^{D-2} \ln \left[\left(\mathcal{G}_{q}\left(D, \mu_{j}, \mu_{j+1}\right)\right)^{\frac{1}{1-q}}\left(\mathcal{G}_{p}\left(D, \mu_{j}, \mu_{j+1}\right)\right)^{\frac{1}{1-p}}\right]+\ln 4
\end{aligned}
$$

with $\frac{1}{p}+\frac{1}{q}=2[64]$. When the spatial dimension is $D=3$ this expression boils down to

$$
\begin{aligned}
& R_{q}\left[\rho_{n, l, m}\right]+R_{p}\left[\gamma_{\eta, L,\left\{\mu_{j}\right\}}\right]=3 \ln \left(\frac{\pi}{2}\right)+\frac{2 q}{q-1} \ln [2 n] \\
+ & \ln \left[\mathcal{F}_{q}(3, n, l)^{\frac{1}{1-q}} \overline{\mathcal{F}}_{p}(3, n, l)^{\frac{1}{1-p}} \mathcal{A}_{q}(3, l)^{\frac{1}{1-q}} \overline{\mathcal{A}}_{p}(3, l)^{\frac{1}{1-p}}\right] \\
+ & \ln \left[\left(\mathcal{G}_{q}(3, l, m)\right)^{\frac{1}{1-q}}\left(\mathcal{G}_{p}(3, l, m)\right)^{\frac{1}{1-p}}\right] \\
+ & \ln \left[4\left(\frac{\Gamma(q m+1)}{\Gamma\left(q m+\frac{3}{2}\right)}\right)^{\frac{1}{1-q}}\left(\frac{\Gamma(p m+1)}{\Gamma\left(p m+\frac{3}{2}\right)}\right)^{\frac{1}{1-p}}\right]
\end{aligned}
$$


Finally and most interesting, the expression (41) in the limit $D \rightarrow \infty$ becomes

$$
R_{q}\left[\rho_{\eta, L,\left\{\mu_{j}\right\}}\right]+R_{p}\left[\gamma_{\eta, L,\left\{\mu_{j}\right\}}\right] \sim D \ln \left(2 \pi(2 q)^{\frac{1}{2-2 q}}(2 p)^{\frac{1}{2-2 p}}\right)
$$

which is the saturation value of the Rényi-entropy-based uncertainty relation found independently by Bialynicki-Birula [61] and Zozor-Portesi-Vignat [62, 63].

$$
R_{q}[\rho]+R_{p}[\gamma] \geq D \ln \left(2 \pi(2 q)^{\frac{1}{2-2 q}}(2 p)^{\frac{1}{2-2 p}}\right), \quad \frac{1}{p}+\frac{1}{q}=2 .
$$

This fact is not only a partial checking of our results but also it is in accordance with similar findings obtained in a very different way. 


\section{CONCLUSIONS}

In this work we have explicitly calculated the total position $R_{q}\left[\rho_{n, l,\{\mu\}}\right]$ and momentum $R_{q}\left[\gamma_{n, l,\{\mu\}}\right]$ Rényi entropies (with integer $q$ greater than 1) for all the quantum-mechanically allowed hydrogenic states in terms of the Rényi parameter $q$, the spatial dimension $D$, the nuclear charge $Z$ as well as the hyperquantum numbers, $(n, l,\{\mu\})$, which characterize the corresponding wavefunction of the states. We have learnt for instance that the Rényi entropies behaves as

$D \ln \left(\frac{\pi^{\frac{1}{2}} \eta}{2 Z}\right)$ and $D \ln \frac{Z}{\eta}$ in the position and momentum spaces, respectively, so that the joint Rényi uncertainty measure linearly grows with the space dimensionality and it does not depend on the nuclear charge. Moreover, we have analytically shown the dependence of the Rényi entropies on the hyperquantum numbers, such as we have illustrated for the three-dimensional hydrogenic quasi-circular states; in the latter case we observe, in particular, that the joint position-momentum entropy sum is an increasing function of the quantum numbers.

We have used a recent methodology which allows to determine the involved integral functionals by taking into account the linearization formula and orthogonality conditions of the Laguerre and Jacobi polynomials; the latter ones are closely connected to the Gegenbauer polynomials which control the angular part of the wavefunctions in both conjugated spaces as well as the radial wavefunction in momentum space. The final expressions for the Rényi entropies in position and momentum spaces are expressed in a compact way by use of a multivariate hypergeometric function of Lauricella and Srivastava-Daoust types evaluated at $1 / q$ and unity, respectively; indeed, note that all sums to be evaluated are finite. Moreover, as a byproduct, we have been able to obtain the exact value of the angular contribution for the Rényi entropies of any non-relativistic and spherically-symmetric multidimensional quantum system. Finally, it remains as an open problem the extension of this result to the limiting case $q \rightarrow 1$, which corresponds to the Shannon entropy, and the Rényi entropies for any real value of the parameter $q$. The latter requires a completely different approach, still unknown to the best of our knowledge.

\section{Acknowledgments}

This work has been partially supported by the Projects FQM-7276 and FQM-207 of the Junta de Andalucía and the MINECO-FEDER grants FIS2014- 54497P, FIS2014-59311P and FIS201789349-P. 


\section{References}

[1] Rényi A 1961 Proceedings of the 4th Berkeley Symposium on Mathematical Statistics and Probability; Neyman J, Ed.; University of California Press: Berkeley, CA, USA, 1961; Volume 1, p. 547

[2] Yule G U 1938 Biometrika 301

[3] Sichel H S 1947 J. R. Stat. Soc., Ser. A 110337

[4] Sichel H S 1949 Biometrika 36404

[5] Sen K D (ed.) 2012 Statistical Complexities: Application to Electronic Structure (Berlin: Springer)

[6] Tsallis C 1988 J. Stat. Phys. 52479

[7] Tsallis C 2009 Introduction to Nonextensive Statistical Mechanics. Approaching a Complex World (Berlin: Springer)

[8] Abe S and Okamoto Y 2001 Nonextensive Statistical Mechanics and Its Applications (Berlin: Springer)

[9] Aczel J and Daroczy Z 1975 On Measures of Information and Their Characterizations (New York: Academic Press)

[10] Jizba P and Arimitsu T 2004 Phys. Rev. E 69026128

[11] Jizba P and Arimitsu T 2004 Ann. Phys. 31217

[12] Rosso O A, Martin M T, Figliola A, Keller K and Plastino A 2006 J. Neuroscience Meth. 153163

[13] Brody D C, Buckley I R C and Constantinou I 2007 Phys. Lett. A 366298

[14] Leonenko N, Pronzato L and Savani V 2008 Ann. Stat. 402153

[15] Nielsen F and Nock R 2011 IEEE Trans. Inf. Theory 201

[16] Aptekarev A I, Dehesa J S, Sánchez-Moreno P and Tulyakov D N 2012 J. Math. Chem. 501079

[17] Aptekarev A I, Dehesa J S, Sánchez-Moreno P and Tulyakov D N 2012 Contemp. Math. 57819

[18] Sánchez-Moreno P, Zarzo A, Dehesa J S and Guerrero A 2013 Applied Math. Comput. 22325

[19] Calixto M, Nagy A, Pasarela I and Romera E 2013 Phys. Rev A 85053813

[20] Carrillo J A and Toscani G 2014 Nonlinearity 273159

[21] Dehesa J S, Guerrero A and Sánchez-Moreno P 2015 J. Math. Chem. 53573

[22] Aptekarev A I, Tulyakov D N, Toranzo I V and Dehesa J S 2016 Eur. Phys. J. B 8985

[23] Dehesa J S, López-Rosa S and Manzano D 2012 Entropy and Complexity Analysis of D-Dimension at Quantum Systems Statistical Complexities: Application to Electronic Structure ed K D Sen (Berlin/Heidelberg: Springer)

[24] Bialynicki-Birula I and Rudnicki L 2011 Entropic uncertainty relations in quantum physics Statistical Complexities: Application to Electronic Structure ed K D Sen (Berlin/Heidelberg: Springer)

[25] Jizba P, Dunningham J A and Joo J 2015 Ann. Phys. 35587

[26] Portesi M, Holik F, Lamberti P W, Bosyk G M, Bellomo G, and Zozor S 2018 Eur.Phys. J.: Special Topic (to appear). 
[27] Dehesa J S,Toranzo I V and Puertas-Centeno D 2017 Int. J. Quantum Chem. 11748

[28] Puertas-Centeno D, Toranzo I V and Dehesa J S 2018 Eur. Phys. J.-Special Topic (to appear)

[29] Francini F, Its A R and Korepin V E 2008 J. Phys. A: Math. Theor. 41025302

[30] Calabrese P and Cardy J 2009 J. Phys. A: Math. Theor. 42504005

[31] Calabrese P, Mintchev M and Vicari E 2011 J. Stat. Mech. P09028

[32] Wood A J, Blythe R A and Evans M R 2017 J. Phys. A: Math. Theor. 50475005

[33] Dehesa J S, López-Rosa S, Martínez-Finkelshtein A and Yáñez R J 2010 Int. J. Quant. Chem. 110 1529

[34] Puertas-Centeno D, Temme N, Toranzo I V and Dehesa J S 2017 J. Math. Phys. 58103302

[35] Toranzo I V, Puertas-Centeno D and Dehesa J S 2016 Physica A 4621197

[36] Nieto M M 1979 Am. J. Phys. 47(12) 1067

[37] Yáñez R J, Van Assche W and Dehesa J S 1994 Phys. Rev. A 504

[38] Kostelecky V A and Russell N 1996 J. Math. Phys. 372166

[39] Aquilanti V, Cavalli S and Coletti C 1997 Chem. Phys. 2141

[40] Coletti C, Calderini D and Aquilanti V 2013 Adv. Quantum Chem. 6773

[41] Caruso F, Martins J and Oguri V 2013 Phys. Lett. A 377694

[42] Bures M 2015 Quantum Physics with Extra Dimensions. Doctoral dissertation (Brno). See chapter 9.

[43] Harrison P 2005 Quantum Wells, Wires and Dots: Theoretical and Computational Physics of Semiconductors Nanostructures (New York: Wiley-Interscience).

[44] Li S S and Xia J B 2007 Phys. Lett. A 366120

[45] Nieto M M 2000 Phys. Rev. A 61034901

[46] Dykman M I, Platzman P M and Seddigard P 2003 Phys. Rev. B 67155402

[47] Amelino-Camelia G and Kowaslki-Glikman J 2005 Planck Scale Effects in Astrophysics and Cosmology (Berlin: Springer)

[48] Witten E 1980 Phys. Today 3338

[49] Itzykson C and Zuber J B 2006 Quantum Field Theory (New York: Dover)

[50] Dong S H 2011 Wave Equations in Higher Dimensions (Berlin: Springer Verlag)

[51] Aquilanti V and Avery J 2001 Adv. Quantum Chem. 7139

[52] Coletti C, Calderini D and Aquilanti V 2013 Adv. Quantum Chem. 6773

[53] Toranzo I V and Dehesa J S 2016 Eur. Phys. Lett. (EPL) 11348003

[54] Temme N M, Toranzo I V and Dehesa J S 2017 J. Phys. A: Math. Gen. 50215206

[55] Srivastava H M 1988 Astrophys. Space Sci. 150251

[56] Srivastava H M and Niukkanen A W 2003 Math. Comput. Model. 37245

[57] Niukkanen A W 1985 J. Phys. A: Math. Gen. 18, 1399-1417.

[58] Nikiforov F, Uvarov V B 1988 Special Functions in Mathematical Physics (Basel: Birkhauser-Verlag)

[59] Olver F W J, Lozier D W, Boisvert R F and Clark C W 2010 NIST Handbook of Mathematical Functions (New York: Cambridge University Press) 
[60] Srivastava H M, Karlsson P W 1985 Multiple Gaussian Hypergeometric Series (New York: John Wiley and Sons)

[61] Bialynicki-Birula I 2006 Phys. Rev. A 74052101

[62] Zozor S and Vignat C 2007 Physica A 375499

[63] Zozor S, Portesi M and Vignat C 2008 Physica A 3874800.

[64] In fact, this is only valid provided the functions $\mathcal{F}_{q}, \overline{\mathcal{F}_{q}}$ and $\mathcal{G}_{q}$ exist for any $p, q \in \mathbb{R}$. 